\title{
DETERMINATION OF ELASTIC CONSTANTS BY MONTE CARLO SIMULATIONS
}

\author{
K. W. WOJCIECHOWSKI ${ }^{\#}$ AND K. V. TRETIAKOV\# \\ \#Institute of Molecular Physics, Polish Academy of Sciences \\ Smoluchowskiego 17/19, 60-179 Poznan, Poland \\ \$InternationalCentre for Theoretical Physics, Trieste, Italy \\ e-mail: kww@man.poznan.pl
}

\begin{abstract}
A B S T RA C T : Simple computational method for studying elastic constants of solids is presented and applied to the case of a f.c.c. tethered crystal. It is shown that the system exhibits negative Poisson's ratio in a range of isotropic tension (negative pressure).
\end{abstract}

The role of elastic constants in the solid state physics is twofold: (a) they provide a link between the mechanical and dynamical behaviour of crystals, and (b) they are a means of probing the interatomic forces [1], As it is well known, only for static crystalline systems the elastic constants can be determined exactly by analytic methods [2]. At non-zero temperatures or/and in the presence of disorder, numerical methods are inescapable, in general.

In the seminal paper, Squire, Holt, and Hoover [3] proposed a Monte Carlo simulation method to compute the elastic constants of model systems. Their method, which uses fluctuations of particle positions in samples of fixed shapes, is however not directly applicable to non-smooth interaction potentials like squarewell or hard-body ones. The latter are important model systems corresponding to the very limit of anharmonicity of intermolecular interactions. Studies of elastic properties of such systems are, at least in principle, easy when fluctuations of the sample shape are allowed. The idea of exploiting fluctuations of the (periodic) box containing the sample comes from Parrinello and Rahman [4] who introduced to the simulations the constant stress tensor [5] to control the deformation (strain) of the sample. Ray and Rahman [6] pointed out that, to be rigorous, one should use the tensor of the thermodynamic tension in place of the stress tensor.

The general strain fluctuation method allows one to determine elastic constants of samples which undergo any affine deformations. When one's interests are restricted to systems at constant pressure, the standard constant pressure Monte Carlo method with variable shape of the periodic box [7] is sufficient to determine the elastic constants. The variable box can be conveniently described by a matrix $\underline{\underline{h}}$, whose columns are formed by components of vectors constituting edges of the parallelepiped which plays the role of the box of periodicity [5]. To avoid the unwanted rotation of the box, the number of the independent components should be restricted to $\Delta=D(D+1) / 2$, where $D$ is the dimensionality of 
the system. The partition function at the constant pressure, p, can be written as: $Z_{N}(p, T)=\int d \underline{\underline{h}}^{\Delta} j(\underline{\underline{h}}) \int d \underline{s}^{N} \exp \left\{\left[-p \operatorname{det}(\underline{\underline{h}})+N \ln [\operatorname{det}(\underline{\underline{h}})]-U_{N}(\underline{\underline{h}} \cdot \underline{\underline{s}})\right] / k T\right\}$,

where (i) in the integration over the components of the periodic box, the restrictions imposed on $\underline{\underline{h}}$ (to reduce the number of the free coordinates) are taken into account, (ii) the integration over all the scaled coordinates of the particles, $\underline{s}_{n} \equiv \underline{\underline{h}}^{-1} \underline{\boldsymbol{r}}_{n}$ (the vector $\underline{\boldsymbol{r}}_{n}$ defines coordinates of the particle $n, n=1, \ldots, N$ and $\underline{\underline{h}}^{-1}$ is the inverse of $\left.\underline{\underline{h}}\right)$ is performed within the unit cube, (iii) $j(\underline{\underline{h}})$ is the Jacobian of the mapping transforming the matrix $\underline{\underline{h}}$ into the strain tensor, $\underline{\underline{\epsilon}}$, calculated with respect to the equilibrium state at $p$, (iv) and $U N$ is the potential energy of the system.

In this report we present results of constant pressure simulations of the f.c.c. tethered crystal. The interaction potential of this tethered crystal is equal to zero if the distance between the interacting ${ }^{1}$ particles does not exceed unity, and infinity otherwise. Tethered solids, introduced by Kantor, Kardar and Nelson [8] to model properties of two dimensional elastic membranes, are the simplest, purely entropic models stable in a range of negative pressures. Recently, it has been shown [9] that, in a range of uniform isotropic tensions (negative pressures), two dimensional tethered solids of hexagonal lattice exhibit negative Poisson's ratio, $\nu<0$. It has been also pointed out [10], using exact arguments and free volume approximation, that such a property should occur at any dimensionality not less than two, both in harmonic and tethered solids. The harmonic and tethered solids represent the opposite 'poles' of the elasticity. The first (harmonic solid) corresponds to purely energetic mechanism, and the second (tethered solid) is governed by the entropy alone. Hence, one can expect that negative values of $\nu$ are characteristic for a broad class of systems under uniform tension. Such a general mechanism leading to negative Poisson's ratio is in contrast to the mechanisms known before, which required either special structure of the system [11-14] or special form of the intermolecular interactions [15-18].

System of $\mathrm{N}=108$ particles was studied at various negative pressures. Some runs were also performed for $N=864$ particles to test any number dependence of the results. Typical runs consisted of $5 \times 10^{6}$ cycles (trial steps per particle), i.e. were by about two orders of magnitude longer than typical runs necessary to determine the equation of state of the system with similar accuracy. The elastic constants were computed using the formula:

$$
S_{i j}^{*}=k_{B} T S_{i j}=V_{p}<\Delta \epsilon_{i} \Delta \epsilon_{j}>,
$$

${ }^{1}$ To avoid a collapse of the system, each particle can interact only with specified particles; the interacting particles form a network. 
where $S_{i j}{ }^{*}$ is the tensor of the elastic compliances, $V_{p}$ is the average volume of the system at the pressure $p, T$ is the temperature, the Boltzmann constant, $\Delta \epsilon_{i}=\epsilon_{i}-\left\langle\epsilon_{i}\right\rangle$, and $\langle\ldots\rangle$ means the thermodynamic averaging. The Voigt convention, $\epsilon_{x x} \rightarrow \epsilon_{1}, \ldots, \epsilon_{y z} \rightarrow \epsilon_{6} / 2$, was used for the components of the strain and compliance tensors.

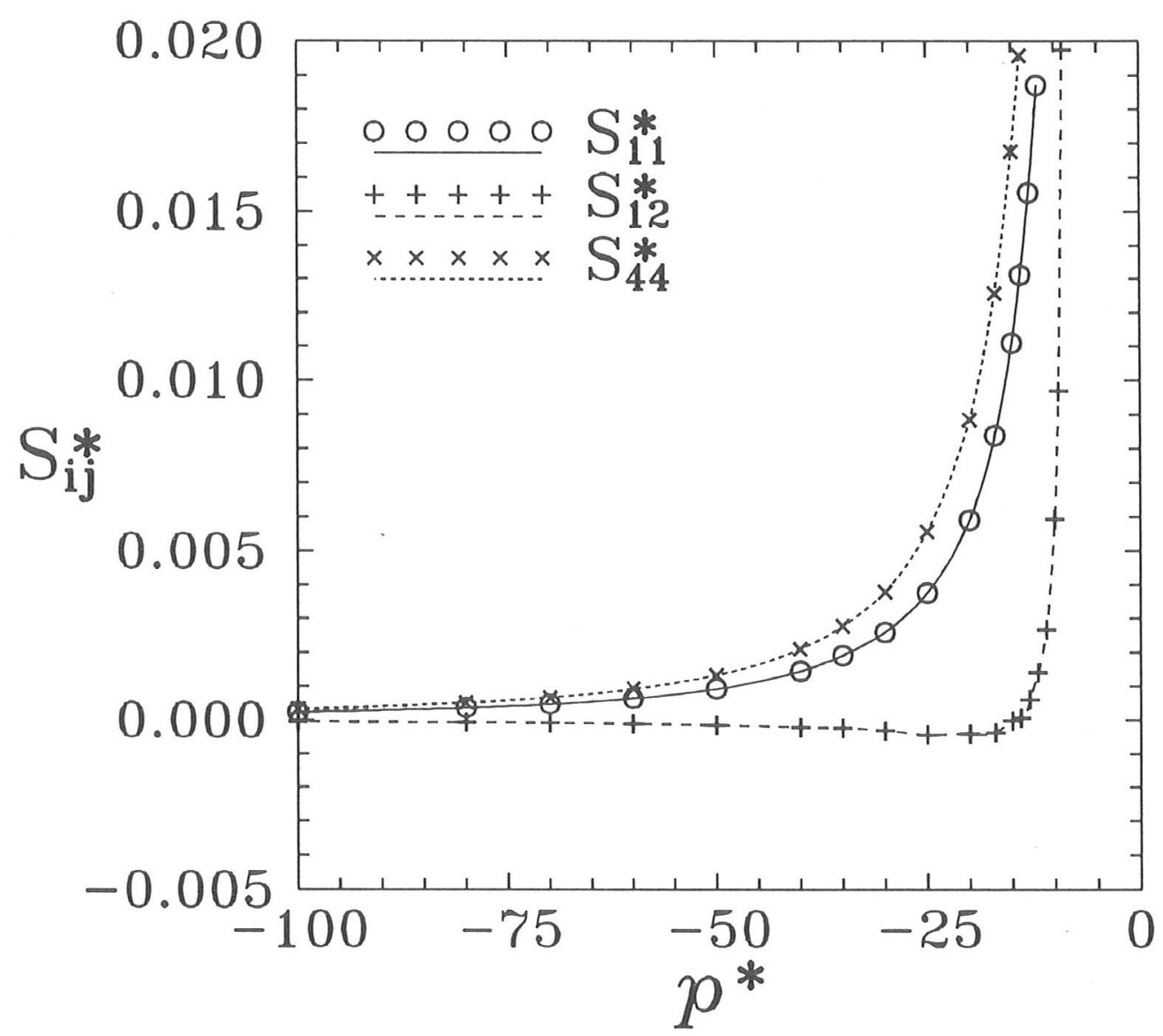

Fig.l. The compliances, $S_{i j}^{*}$ of the f.c.c. tethered crystal versus the reduced pressure, $p^{*}=p / k_{B} T$.

Pressure dependence of the compliances is shown in Fig.1. It can be seen there that $S_{12}^{*}$ changes sign in the vicinity of $\mathrm{p}=14$. This means that the Poisson's ratio:

$$
\nu=-\frac{S_{12}^{*}}{S_{11}^{*}},
$$

changes sign also, as shown in Fig.2. 


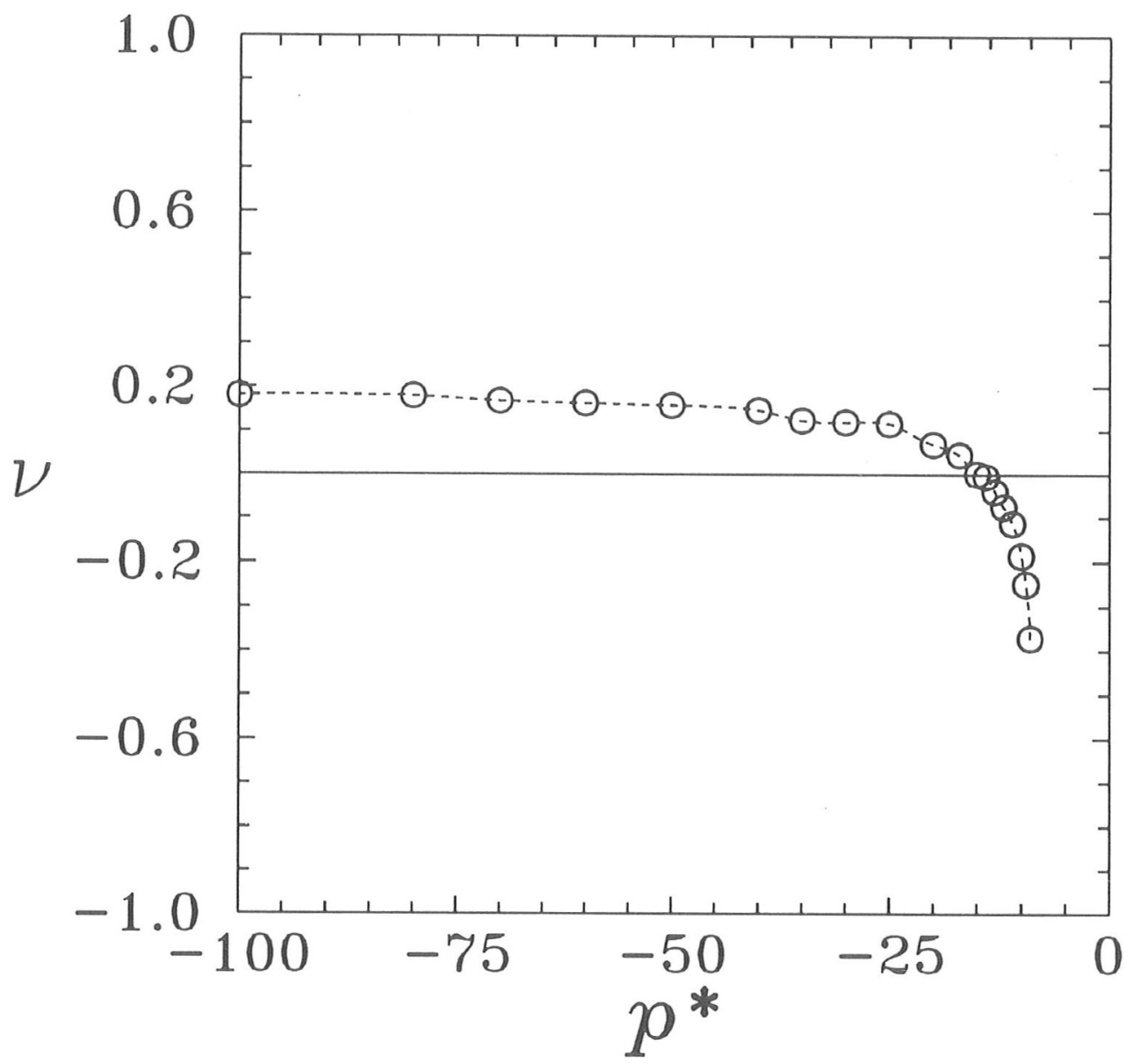

Fig.2. The Poisson's ratio of the f.c.c. tethered crystal versus the reduced pressure, $p^{*}=p / k_{B} T$.

The present simulations confirm the theoretical prediction, by the free volume approximation [10], that the three dimensional tethered solid should exhibit negative Poisson's ratio. This fact, combined with the rigorous results available for harmonic crystals [10], suggests that in a range of negative pressures various systems should exhibit $\nu<0$. Systems of negative Poisson's ratios exhibit many surprising and useful properties [19]. In such a context, the general mechanism leading to negative Poisson's ratios, described in this report, can be interesting from the point of view of practical application.

Acknowledgments: This work was supported by the grant $8 \mathrm{~T} 11 \mathrm{~F} 01008 \mathrm{p} 04$ of the Polish Committee for Scientific Research (KBN). Computer simulations were performed at Poznań Computer and Networking Centre (PCSS). One of the au- 
thors (K.W.W.) is grateful to Professor Yu Lu for invitation to the Condensed Matter Group at the International Centre for Theoretical Physics.

(Received June 22, 1995)

\section{References}

[1] M. Born and K. Huang, Dynamical Theory of Crystal Lattices (Clarendon, Oxford, 1954).

[2] J. H. Weiner, Statistical Mechanics of Elasticity (Wiley, New York, 1983).

[3] D. R. Squire, A. C. Holt, and W. G. Hoover, Physica 42, 388 (1968).

[4] M. Parrinello and A. Rahman, J. Chem. Phys. 76, 2662 (1982).

[5] M. Parrinello and A. Rahman, J. Appl. Phys. 52, 7182 (1981).

[6] J. R. Ray and A. Rahman, J. Chem. Phys. 80, 4423 (1984).

[7] K. W. Wojciechowski, A. C. Brańka, and M. Parrinello, Molec. Phys. 53, 1541 (1984).

[8] Y. Kantor, M. Kardar, and D. R. Nelson, Phys. Rev. Lett. 57, 791 (1986);

Y. Kantor, M. Kardar, and D. R. Nelson, Phys. Rev. A35, 3056 (1987).

[9] D. H. Boal, U. Seifert, and J. C. Schillcock, Phys. Rev. E48, 4274 (1993).

[10] K. W. Wojciechowski, Mol. Phys. Reports 10 (1995).

[11] R. F. Almgren, Journal of Elasticity 15, 427 (1985).

[12] R. Lakes, Science 235, 1038 (1987).

[13] B. D. Caddock and K. E. Evans, J. Phys. D22, 1877 (1989).

[14] G. W. Milton, J. Mech. Phys. Solids 40, 1105 (1992).

[15] K. W. Wojciechowski, Mol. Phys. 611247 (1987);

K. W. Wojciechowski and A. C. Brańka, Phys. Rev. A40, 7222 (1989).

[16] R. J. Bathurst and L. Rothenburg, J. Appl. Mechanics. 55, 17 (1988);

R. J. Bathurst and L. Rothenburg, Int. J. Eng. Sei. 26, 373 (1988).

[17] K. W. Wojciechowski, Phys. Lett. 137, 61 (1989).

[18] L. Rothenburg, A. A. Berlin, and R. J. Bathurst, Nature 354, 470 (1991).

[19] K. W. Wojciechowski and A. C. Brańka, Mol. Phys. Reports 6, 71 (1994) and references therein. 